\title{
Review: group-based education in self management strategies improves outcomes in type 2 diabetes mellitus
}

Deakin T, McShane CE, Cade JE, et al. Group based training for self-management strategies in people with type 2 diabetes mellitus. Cochrane Database Syst Rev 2005;(2):CD003417.

Clinical impact ratings Endocrine $\star \star \star \star \star \star \star$ is

In patients with type 2 diabetes mellitus, does group-based education (GBE) in self management improve clinical, lifestyle, and psychosocial outcomes?

\section{METHODS}

Data sources: electronic databases that included the Cochrane
Library, Medline, CINAHL, ERIC, ASSIA, AMED, PsycINFO, and
EMBASE/Excerpta Medica; bibliographies of relevant articles;
and contact with experts in the field.
Study selection and assessment: randomised controlled trials
(RCTs) or observational studies that compared GBE (delivered in
primary or secondary care, based on learner/patient centred
education, $\geqslant 6$ participants in a group with $\geqslant 1$ session) with a
control condition that included usual care, no intervention, or a
waiting list.
Outcomes: clinical (glycated haemoglobin, fasting glucose
concentration, and medication use), lifestyle (diabetes
knowledge), and psychosocial (quality of life empowerment/self-
efficacy).

\section{MAIN RESULTS}

8 RCTs $(n=1260)$ and 3 observational studies $(n=272)$ met the selection criteria. Random effects meta-analyses showed that glycated haemoglobin and fasting glucose concentrations were lower in the intervention group than in the control group (table) and that diabetes knowledge scores were greater in the intervention group than in the control group (standardised mean difference $0.95,95 \%$ CI 0.72 to 1.18 ) ( 3 trials, $\mathrm{n}=432$ ). More patients in the intervention group than in the control group reduced their use of diabetes medication over 12-14 months (relative benefit increase $825 \%$, CI 202 to 2738) (5 trials, $\mathrm{n}=654)$. l RCT $(\mathrm{n}=314)$ assessing empowerment and psychosocial self efficacy reported greater total empowerment scores in the intervention group than in the control group throughout follow up ( $\mathrm{p}$ value $<0.05$ )

\section{CONCLUSIONS}

In patients with type 2 diabetes mellitus, group-based education in self management strategies improves clinical and lifestyle outcomes.

\section{A modified version of this abstract appears in Evidence-Based Nursing.}

For correspondence: Dr T Deakin, Burnley General Hospital, Burnley, UK. trudi.deakin@nhs.net

Sources of funding: National Health Service (Executive) North West UK and British Dietetic Association UK.

\section{Commenfary}

D eakin et al concluded that GBE supporting patient self management improves patient knowledge and clinical outcomes. The 11 studies in the systematic review included GBE in community and medical practice settings.

Community GBE often includes a heterogeneous population and diverse implementation strategies. Successful translation of this model depends on a community intervention site with professionals and community workers providing culturally relevant activities. ${ }^{\text {' In a }}$ community GBE strategy, for example, a facilitator invites patients to reflect on self management experiments and to set their own goals. ${ }^{2}$ This approach emphasises that the acquisition of knowledge (the traditional outcome of education) and lifestyle changes are not equivalent outcomes; more knowledge is not sufficient for the successful adoption of a healthy lifestyle.

GBE in medical practice is often used in combination with individual office visits. This combination has been shown to improve independence, functional status, health related quality of life, and healthcare use. ${ }^{3}$ During GBE, patients participate in an organised warm up period, receive multidisciplinary team presentations on specific health topics, and take part in group discussions. Patients then receive individualised care. ${ }^{4}$

It is time that health systems adopt new strategies for patient self management support that extend beyond traditional one-on-one clinical encounters. In the right context and with appropriate patients, evidence supports the value of GBE in providing knowledge as well as goal setting and problem solving for physical activity, nutrition, medication adherence, and behaviour change. Susan S Bjornsen, RN, CDE
Steven A Smith, MD
Mayo Clinic College of Medicine
Rochester, Minnesota, USA

1 Brown SA, Garcia AA, Kouzekanani K et al. Culturally competent diabetes self-management education for Mexican Americans: the Starr County border health initiative. Diabetes Care 2002;25:259-68.

2 Funnell MM, Nwankwo R, Gillard ML, et al. Implementing an empowerment-based diabetes self-management education program. Diabetes Educ 2005;31:53-61.

3 Noffsinger EB, Scott JC. Understanding today's group visit models. Group Pract J 2000:48:46-58.

4 Scott JC, Conner DA, Venohr I, et al. Effectiveness of a group outpatient visit model for chronically ill older health maintenance organization members: a 2-year randomized trial of the cooperative health care clinic. J Am Geriatr Soc 2004;52:1463-70.

5 Lorig KR, Ritter PL, Jacquez A. Outcomes of border health Spanish/English chronic disease self-management programs. Diabetes Educ 2005;31:4019

Group-based education (GBE) in self management strategies $v$ a control condition that included usual care, no intervention, or a waiting list in type 2 diabetes mellitus*

\begin{tabular}{|c|c|c|c|c|c|}
\hline \multirow[b]{2}{*}{ Outcomes } & \multirow[b]{2}{*}{ Follow up in months } & \multirow[b]{2}{*}{ Number of trials ( $\mathrm{n}$ ) } & \multicolumn{2}{|c|}{ Weighted means } & \multirow[b]{2}{*}{ Weighted mean difference $(95 \% \mathrm{Cl}$} \\
\hline & & & $\overline{\mathrm{GBE}}$ & Control & \\
\hline \multirow[t]{3}{*}{ Glycated haemoglobin (\%) } & $4-6$ & $3(395)$ & 9.53 & 10.88 & $-1.35(-1.93$ to -0.78$)$ \\
\hline & $12-14$ & 7 (1044) & 7.93 & 8.75 & $-0.82(-0.99$ to -0.65$)$ \\
\hline & 24 & 2 (333) & 6.54 & 7.51 & $-0.97(-1.40$ to -0.54$)$ \\
\hline Fasting blood glucose $(\mathrm{mmol} / \mathrm{l})$ & $12-14$ & $4(641)$ & 9.55 & 10.72 & $-1.17(-1.63$ to -0.72$)$ \\
\hline
\end{tabular}

